This item was submitted to Loughborough's Research Repository by the author.

Items in Figshare are protected by copyright, with all rights reserved, unless otherwise indicated.

\title{
Bounds for the mixing parameter within the CC-CMA algorithm applied in non ideal multiuser environments
}

PLEASE CITE THE PUBLISHED VERSION

PUBLISHER

(c) IEEE

VERSION

VoR (Version of Record)

\section{LICENCE}

CC BY-NC-ND 4.0

\section{REPOSITORY RECORD}

Luo, Yuhui, and Jonathon Chambers. 2019. "Bounds for the Mixing Parameter Within the CC-CMA Algorithm Applied in Non Ideal Multiuser Environments". figshare. https://hdl.handle.net/2134/5822. 
This item was submitted to Loughborough's Institutional Repository (https://dspace.lboro.ac.uk/) by the author and is made available under the following Creative Commons Licence conditions.

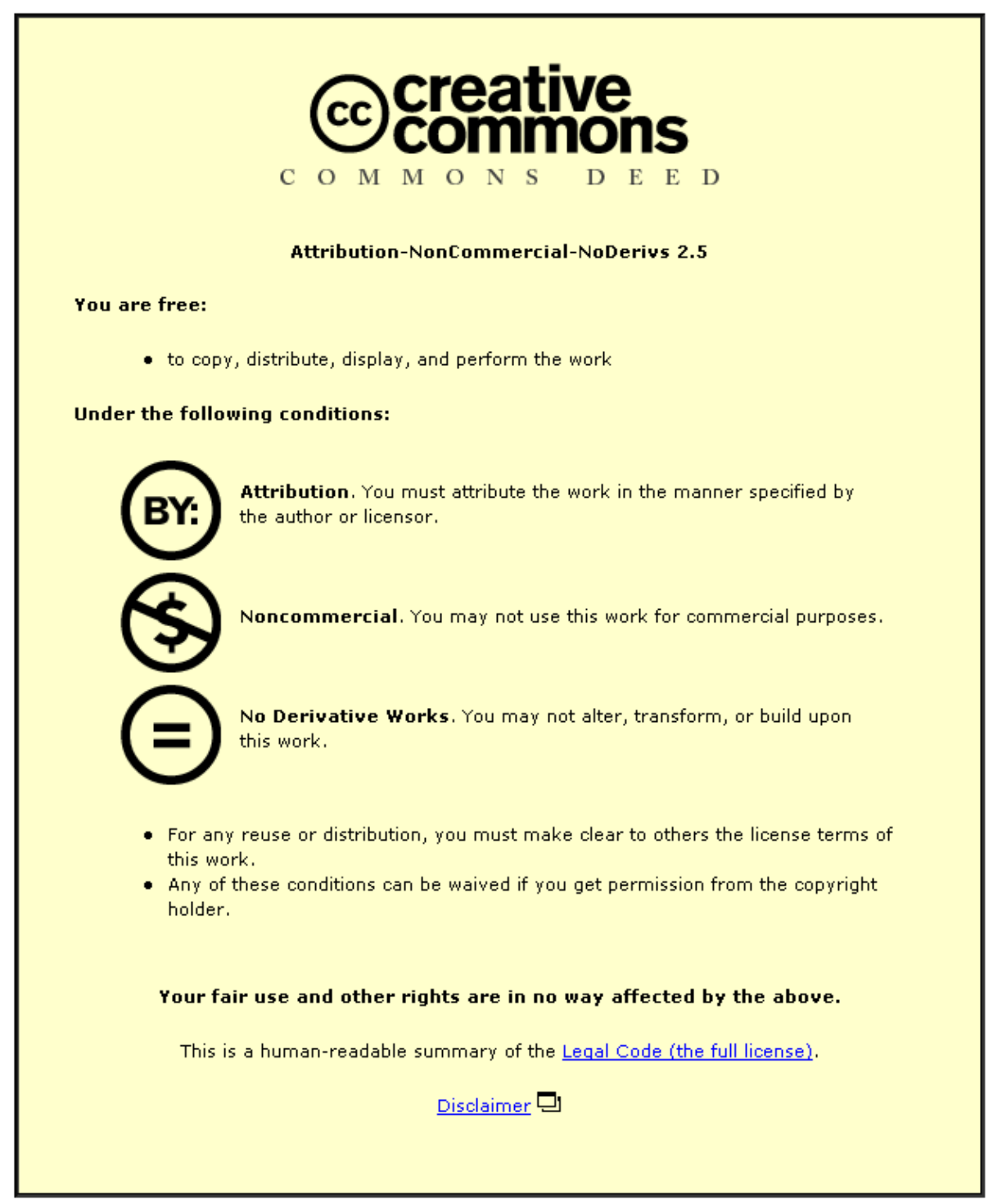

For the full text of this licence, please go to: http://creativecommons.org/licenses/by-nc-nd/2.5/ 


\title{
BOUNDS FOR THE MIXING PARAMETER WITHIN THE CC-CMA ALGORITHM APPLIED IN NON IDEAL MULTIUSER ENVIRONMENTS
}

\author{
Y. Luo ${ }^{1}$ and J.A. Chambers ${ }^{2}$ \\ ${ }^{1}$ Communications and Signal Processing Group,D ept. of Electrical and Electronic Engineering \\ Imperial college of Science,T echnology and Medicine,L ondon,S W7 2BT,U .K. \\ Email: Yuhui.Luo@ic.ac.uk \\ ${ }^{2}$ Dept. of Electronic and Electrical Engineering \\ University of Bath,B ath,B A2 7AY,U .K.
}

\begin{abstract}
We derive new bounds for the mixing parameter, $\gamma$, within the cross-correlation constant modulus algirithm (CC-CMA) for blind source separation and equalization in non-ideal multiuser environments. Channel undermodelling and noise are considered when the complex sources are circularly symmetric. These tighter bounds are obtained by surface topography of the error performance surface of the CC-CMA algorithm, and replace earlier work which suggested that $\gamma>4 / 3$. The validity of the bounds is confirmed by simulation studies.
\end{abstract}

\section{INTRODUCTION}

In multiuser wireless communications, space division multiple access (SDMA) may be exploited when multiple i.i.d. digital signals that originate from spatially separated sources are transmitted through linear channels and picked up by an array of antennas. As the result of multipath propagation and the presence of cochannel system users, the received signals are distorted not only by intersymbol interference (ISI) but also by interuser interference (IUI). Many blind adaptive channel equalization algorithms with implicit signal separation capability have been proposed in this context. Among which, the cross-correlation and constant modulus algorithm (CC-CMA) [5] reconstructs the underlying constant modulus property of communication signals. To prevent repeated retrieval of the same source, a term which penalizes cross-correlation between multiple output signals is introduced. In [3], based on the use of sequential start-up of equalizers, a simplified CC-CMA cost function is proposed, where only the previous retrieved sources are included in the cross-correlation term. Assuming a real system and that the outputs of the previous equalizers are perfect, the authors show that the mixing parameter $\gamma$ should be greater than $4 / 3$ to avoid ill convergence. However, in practice, due to channel undermodelling or noise, perfect equaliza- tion is not always possible. Meanwhile communication signals are generally complex rather than real. Therefore we relax the perfect equalization condition and, by studying the surface topography of the CC-CMA cost function, we obtain a tighter bound for the mixing parameter in a complex system.

\section{CC-CMA ALGORITHM}

We assume a $d$ user and $r$ antenna system. The multiple antenna output is processed with $d$ parallel space-time equalizers to retrieve all the sources. Notations $(\cdot)^{H},(\cdot)^{T}$ and $(\cdot)^{*}$ denote respectively hermitian, transpose and complex conjugate. The orders of the channela nd the sub-equalizer are respectively $M$ and $N$. The $i^{\text {th }}$ source signal at time $k$ is written as $\mathbf{s}_{i}(k)=\left[s_{i}(k) \ldots s_{i}(k-M-N)\right]^{T}$ and the source vector fora 11 sources is represented by $\mathbf{s}(k)=$ $\left[\mathbf{s}_{1}^{T}(k) \ldots \mathbf{s}_{d}^{T}(k)\right]^{T}$. Let $\mathbf{x}_{j}(k)=\left[x_{j}(k) \ldots x_{j}(k-N)\right]^{T}$ be the $j^{\text {th }}$ antenna output vector. The space-time equalizer regressor is $\mathbf{x}(k)=\left[\mathbf{x}_{1}^{T}(k) \ldots \mathbf{x}_{r}^{T}(k)\right]^{T}$. Defining $\Delta^{T}$ as the channel convolution matrix, we obtain $\mathbf{x}(k)=\triangle^{T} \mathbf{s}(k)$. Describe the $l^{t h}$ space-time equalizer tap vector as $\mathrm{w}_{l}(k)$. Its output is $y_{l}(k)=\mathbf{w}_{l}^{T}(k) \mathbf{x}(k)=\mathbf{h}_{l}^{T}(k) \mathbf{s}(k)$, where $\mathbf{h}_{l}(k)=\Delta \mathbf{w}_{l}(k)$ is the combined channel $+l^{\text {th }}$ equalizer impulse response. The CC-CMA algorithm cost function for the $l^{\text {th }}$ equalizer is written as

$$
\begin{aligned}
J_{l}(k)= & E\left\{\left(\left|y_{l}(k)\right|^{2}-R_{2}\right)^{2}\right\} \\
& +\gamma \sum_{j=1}^{l-1} \sum_{\delta=-(M+N)}^{M+N}\left|E\left\{y_{l}(k) y_{j}^{*}(k-\delta)\right\}\right|^{2}(1)
\end{aligned}
$$

where $E\left\{\left(\left|y_{l}(k)\right|^{2}-R_{2}\right)^{2}\right\}$ is the constant modulus cost, $\sum_{\delta} \sum_{j}\left|E\left\{y_{l}(k) y_{j}^{*}(k-\delta)\right\}\right|^{2}$ is the cross-correlation cost between the $l^{\text {th }}$ equalizer and the retrieved sources, $R_{2}=$ $\frac{E\left\{|s|^{4}\right\}}{E\left\{|s|^{2}\right\}}$ is the so-called dispersion constant and $\gamma \in R^{+}$is the mixing parameter. 


\section{SURFACE ANALYSIS}

It can be observed from eq (1) that the accuracy of the retrieved sources affects the convergence of the current equalizer. In practice, due to hardware constraints on equalizer length and channel noise, residual error at the equalizer output is unavoidable. Therefore we model the previous equalizer output ast he retrieval of a particular source symbol with possible delay, together with residual ISI and IUI. For clarity, we present the deviation for two users. But similar deviation can be extended to more users. Denoting $p_{1}=$ $M+N, p=2(M+N)+1, \mathbf{h}_{1}=\left[\alpha_{1} \ldots \alpha_{p_{1}} \beta_{p_{1}+1} \ldots \beta_{p}\right]^{T}$ and $\mathbf{h}_{2}=\left[h_{0} h_{1} \ldots h_{p}\right]^{T}$. Without loss ofg enerality, we assume that the first equalizer reconstructss ource-1, i.e., $\left|\alpha_{d}\right|>\left|\alpha_{i}\right|, i \neq d$ and $d \in\left(0, p_{1}\right]$. The first equalizer output is given by

$$
y_{1}(k)=\alpha_{d} s(k, d)+\sum_{i=0, i \neq d}^{p_{1}} \alpha_{i} s(k, i)+\sum_{i=p_{1}+1}^{p} \beta_{i} s(k, i)
$$

where $s(k, i)$ is the $i^{\text {th }}$ elemento $\mathrm{f}$ the vector $\mathbf{s}(k)$. The coefficients $\left\{\alpha_{i}, i \neq d, i \in\left(0, p_{1}\right]\right\}$ and $\left\{\beta_{i}, i \neq d, i \in\right.$ $\left.\left(p_{1}, p\right]\right\}$ respectively contribute to the residual ISI and IUI.

With respect to eq (1), thec ross-correlation cost for equalizer-2 is written as $\sum_{\delta=-(N+M)}^{N+M}\left|E\left\{y_{2}(k) y_{1}^{*}(k-\delta)\right\}\right|^{2}$. We replace $y_{1}^{*}(k-\delta)$ with the expression shown in eq (2). Using the independent property of the sources and considering that the cross terms $\left(\sum_{\delta} \sum_{i} \sum_{j, i \neq j} \alpha_{i} \alpha_{j}^{*} h_{i+\delta}^{*} h_{j+\delta}\right)$, $\left(\sum_{\delta} \sum_{i} \sum_{j, i \neq j} \beta_{i} \beta_{j}^{*} h_{i+\delta}^{*} h_{j+\delta}\right),\left(\sum_{\delta} \sum_{i} \sum_{j} \alpha_{i} \beta_{j}^{*} h_{i+\delta}^{*} h_{j+\delta}\right)$ and $\left(\sum_{\delta} \sum_{i} \sum_{j} \alpha_{i}^{*} \beta_{j} h_{i+\delta} h_{j+\delta}^{*}\right)$ are small value compared with the squared terms $\sum_{i=0}^{p_{1}}\left|\alpha_{i}\right|^{2} \sum_{j=0}^{p 1}\left|h_{j}\right|^{2}$ and $\sum_{i=0}^{p}\left|\beta_{i}\right|^{2} \sum_{j=p 1+1}^{p}\left|h_{j}\right|^{2}$ and therefore can be neglected, as [4], the decorrelation cost is approximated by

$$
\begin{aligned}
& \sum_{\delta=-(N+M)}^{N+M}\left|E\left\{y_{2}(k) y_{1}^{*}(k-\delta)\right\}\right|^{2} \\
\approx & \sigma_{s}^{4}\left(\theta_{1} \sum_{j=0}^{p_{1}}\left|h_{j}\right|^{2}+\theta_{2} \sum_{j=p_{1}+1}^{p}\left|h_{j}\right|^{2}\right)
\end{aligned}
$$

where forn otational convenience $\theta_{1}=\sum_{i=0}^{p_{1}}\left|\alpha_{i}\right|^{2}, \theta_{2}=$ $\sum_{i=p_{1}+1}^{p}\left|\beta_{i}\right|^{2}$ and $\sigma_{s}^{2}$ is the signal variance. Notice that as source- 1 is assumed to be reconstructed, with very high probability the energy contribution of source-1 isg reater than that from source-2 at the equalizer-1 output, i.e., $\sum_{i=0}^{p_{1}}\left|\alpha_{i}\right|^{2}>\sum_{i=p_{1}+1}^{p}\left|\beta_{i}\right|^{2}$, or equivalently $\theta_{1}>\theta_{2}$.

Combining the expression of constant modulus cost given in [1], the costf unction for the second equalizer $J_{2}$ is written as

$$
J_{2}=k_{s} \sigma_{s}^{4} \sum_{i=0}^{p}\left|h_{i}\right|^{4}+2 \sigma_{s}^{4} \sum_{i=0}^{p} \sum_{j=0, i \neq j}^{p}\left|h_{i}\right|^{2}\left|h_{j}\right|^{2}
$$

$$
\begin{aligned}
& +\left|E\left\{s^{2}(k)\right\}\right|^{2} \sum_{i=0}^{p} \sum_{j=0, i \neq j}^{p} h_{i}^{2}\left(h_{j}^{*}\right)^{2}-2 k_{s} \sigma_{s}^{4}\left\|\mathbf{h}_{2}\right\|_{2}^{2} \\
& +k_{s} \sigma_{s}^{4}+\gamma \sigma_{s}^{4}\left(\theta_{1} \sum_{j=0}^{p 1}\left|h_{j}\right|^{2}+\theta_{2} \sum_{j=p 1+1}^{p}\left|h_{j}\right|^{2}\right)
\end{aligned}
$$

where $k_{s}=\frac{E\left\{|s(k)|^{4}\right\}}{\sigma_{s}^{4}}$ is the source kurtosis and $\|\bullet\|_{2}$ is the two-norm of the vector. In the complex case, as in [2], we study signal constellations that satisfy the circularity condition $E\left\{s(k)^{2}\right\}=0$, which holds for most constellations.

Lemma 1 1. A necessary condition on $\gamma$ for the elimination of undesirable minima in a two-user scheme is

$$
\frac{\left(2-k_{s}\right) 2 k_{s}}{2 \theta_{1}-k_{s} \theta_{2}} \leq \gamma<\frac{2 k_{s}}{\theta_{2}}
$$

Proof outline: Given $E\left\{s(k)^{2}\right\}=0$, the gradient of eq (4) is given by

$$
\mathbf{g}_{l}(k)=2 \Lambda \mathbf{h}_{2}
$$

where $\Lambda=\operatorname{diag}\left(\Lambda_{0}+\frac{\gamma}{2} \theta_{1}, \ldots, \Lambda_{p}+\frac{\gamma}{2} \theta_{1}, \Lambda_{p_{1}+1}+\frac{\gamma}{2} \theta_{2}, \ldots\right.$ $\left.\Lambda_{p}+\frac{\gamma}{2} \theta_{2}\right), \Lambda_{l}=\sigma_{s}^{4}\left(\left|h_{l}\right|^{2}\left(k_{s}-2\right)+2\left\|\mathbf{h}_{2}\right\|_{2}^{2}-k_{s}\right)$. The diagonal of the Hessian matrix is

$$
\begin{aligned}
H_{l, l}= & 2 \sigma_{s}^{4}\left(2\left(k_{s}-1\right)\left|h_{l}\right|^{2}+2\left\|\mathbf{h}_{2}\right\|_{2}^{2}-k_{s}\right) \\
& +\sigma_{s}^{4} \begin{cases}\gamma \theta_{1} & 0 \leq l \leq p_{1} \\
\gamma \theta_{2} & p_{1}+1 \leq l \leq p\end{cases}
\end{aligned}
$$

The off-diagonal terms are

$$
H_{l, m}=4 \sigma_{s}^{4} h_{l} h_{m}^{*} \text { and } H_{l, m}=H_{m, l}^{*}
$$

We consider the property of the following classes of stationary points.

(1) $\mathbf{h}_{2}=0$.

With respect to eq (6) and eq (7), the Hessian of this type of stationary point is $H=2 \sigma_{s}^{4} \operatorname{diag}\left(\frac{\gamma}{2} \theta_{1}-k_{s}, \ldots, \frac{\gamma}{2} \theta_{1}-\right.$ $k_{s}, \frac{\gamma}{2} \theta_{2}-k_{s}, \ldots, \frac{\gamma}{2} \theta_{2}-k_{s}$ ). As the equalizer-2 gives trivial output, positive definite Hessian matrix is undesirable. Therefore the condition on $\gamma$ is

$$
\gamma<\frac{2 k_{s}}{\theta_{2}}
$$

(2) One $h_{i} \neq 0, i \in\left(0, p_{1}\right]$ and others zeros.

To zero the gradient, according to eq (5), the non-zero $h_{i}$ satisfies $\left|h_{i}\right|^{2}=1-\frac{\gamma \theta_{1}}{2 k_{s}}$. The Hessian is a diagonal matrix with three values, i.e.,

$H_{l, l}=2 \sigma_{s}^{4}\left\{\begin{array}{l}2-k_{s}+\gamma \theta_{1}\left(0.5-1 / k_{s}\right) 0 \leq l \leq p_{1}, l \neq i \\ k_{s}-\frac{\gamma}{2} \theta_{1} \\ 2-k_{s}-\gamma\left(\theta_{1} / k_{s}-0.5 \theta_{2}\right) \quad p_{1}+1 \leq l \leq p\end{array}\right.$

Since this kind of stationary point indicates the repeated retrieval of source-1, we don't expect this stationary point to be a minimum. Hence the condition on $\gamma$ is

$$
\gamma \geq \frac{\left(2-k_{s}\right) 2 k_{s}}{2 \theta_{1}-k_{s} \theta_{2}}
$$


where we assume the source is sub-Gaussian, i.e., $k_{s}<k_{g}$ and $k_{g}=2$ for a complex-valued Gaussian process. This assumption is, $\mathrm{i}$ fact, a condition for perfect equalization.

(3) One $h_{i} \neq 0, i \in\left(p_{1}, p\right]$ and others zeros.

To zero the gradient, the non-zero $h_{i}$ satisfies $\left|h_{i}\right|^{2}=$ $1-\frac{\gamma \theta_{2}}{2 k_{s}}$. The Hessian is a diagonal matrix of three values, i.e.,

$$
H_{l, l}=2 \sigma_{s}^{4}\left\{\begin{array}{l}
2-k_{s}+\gamma\left(\theta_{1} / 2-\theta_{2} / k_{s}\right) \quad 0 \leq l \leq p_{1} \\
k_{s}-\frac{\gamma}{2} \theta_{2} \quad p_{1}+1 \leq l \leq p, l=i \\
2-k_{s}+\gamma \theta_{2}\left(0.5-1 / k_{s}\right) \\
p_{1}+1 \leq l \leq p, l \neq i
\end{array}\right.
$$

As the stationary point indicates the retrieval of source-2, which is the desirable source, positive definite Hessian is desired. The condition on $\gamma$ is

$$
\gamma<\frac{2 k_{s}}{\theta_{2}}
$$

Notice that this is the same condition as eq (8).

(4) $h_{i} \neq 0$ for $v$ values of $i, v>1$ and $i \in\left[0, p_{1}\right]$ and all others are zeros.

Zeroing the gradient yields $\left|h_{i}\right|^{2}=\frac{2\left\|\mathbf{h}_{2}\right\|_{2}^{2}-k_{s}+0.5 \gamma \theta_{1}}{2-k_{s}}$. Since the right-hand side oft he equation is a constant, all non-zero $h_{i}$ s have equal magnitude and we obtain $\left|h_{i}\right|^{2}=$ $\frac{k_{s}-0.5 \gamma \theta_{1}}{k_{s}+2(v-1)}$. Let $D$ be a diagonal matrixw ithe lements: $2 \sigma_{s}^{4} \frac{\gamma v\left(\theta_{2}-\theta_{1}\right)+k_{s}\left(2-k_{s}\right)+0.5 \gamma \theta_{2}\left(k_{s}-2\right)}{k_{s}+2(v-1)}$. Denote matrix $Q$ as

$Q=\frac{2 \sigma_{s}^{4}\left(k_{s}-0.5 \gamma \theta_{1}\right)}{k_{s}+2(v-1)}\left[\begin{array}{lll}k_{s} & \ldots & 2 c_{0} c_{p 1} e^{j \Phi_{p}} \\ \vdots & \ddots & \vdots \\ 2 c_{p_{1}} c_{0} e^{-j \Phi_{p}} & \ldots & k_{s}\end{array}\right]$

where $c_{i} \in\{-1,0,1\}$ for $i \in\left[0, p_{1}\right]$. The Hessian matrix in this case can be expressed in the form of $\left[\begin{array}{ll}Q & 0 \\ 0 & D\end{array}\right]$. As $v>1$, there exist principal minors in $Q$ oft he following form

$$
\frac{2 \sigma_{s}^{4}\left(k_{s}-0.5 \gamma \theta_{1}\right)}{k_{s}+2(v-1)}\left[\begin{array}{ll}
k_{s} & 2 c_{i} c_{j} e^{j \Phi_{j}} \\
2 c_{j} c_{i} e^{-j \Phi_{j}} & k_{s}
\end{array}\right]
$$

As $\theta_{1}>\theta_{2}$, provided the sub-Gaussian source condition $\left(k_{s}<2\right)$ holds and the condition stated in eq $(8)$ is satisfied, the determinanto $f$ eq (14) is negative and therefore $Q$ is indefinite. It is concluded that the stationary points are saddles [1].

(5) $h_{i} \neq 0$ for $v$ values of $i, v>1$ and $i \in\left(p_{1}, p\right]$ and all others are zeros.

Similar to case (4), all non-zero $h_{i} \mathrm{~s}$ are of equal magnitude: $\left|h_{i}\right|^{2}=\frac{k_{s}-0.5 \gamma \theta_{2}}{k_{s}+2(v-1)}$. The Hessian matrix is given by $\left[\begin{array}{ll}D & 0 \\ 0 & Q\end{array}\right]$, where $D$ is a diagonal matrix. When the sources are sub-Gaussian $\left(k_{s}<2\right)$ the condition stated in eq (8) is satisfied, it can be proven that $Q$ is indefinite. Hence the stationary points are saddles.

(6) $h_{i} \neq 0$ for $v_{1}$ values of $i, v_{1} \geq 1, i \in\left[0, p_{1}\right]$ and $h_{j} \neq 0$ for $v_{2}$ values of $i, v_{2} \geq 1$ and $i \in\left(p_{1}, p\right]$.

To zero theg radient, then on-zero $h_{j} \mathrm{~s}$ areg iven by $\left|h_{j}\right|^{2}=\frac{k_{s}-0.5 \gamma \theta_{2}-v_{1} \gamma\left(\theta_{2}-\theta_{1}\right) /\left(k_{s}-2\right)}{k_{s}+2\left(v_{1}+v_{2}-1\right)}$. The existence of $\left|h_{j}\right|^{2}$ requires $\gamma<\frac{k_{s}\left(2-k_{s}\right)}{0.5 \theta_{2}\left(2-k_{s}\right)+v_{1}\left(\theta_{1}-\theta_{2}\right)}$. However, since $\frac{k_{s}\left(2-k_{s}\right)}{0.5 \theta_{2}\left(2-k_{s}\right)+v_{1}\left(\theta_{1}-\theta_{2}\right)}<\frac{\left(2-k_{s}\right) 2 k_{s}}{2 \theta_{1}-k_{s} \theta_{2}}$, when eq (10) is satisfied, $\left|h_{j}\right|^{2}<0$ and thus this kind of stationary point does not exist. Although the term stationary points is used in the preceding analysis for the complex case, the minima correspond to a continuum ofm inima that span the complete range $(0,2 \pi)$ of valid phase values [2].

The analysis for a $d>2$ system is analogous to that in the two user case. Write the previous equalizer- $j$ output as $y_{j}(k)=\sum_{i=0}^{d(N+M+1)-1} h_{j, i}(k) s(k, i)$, where $1 \leq j<$ $l-1$. Define the parameters $\sum_{j=1}^{l-1} \sum_{i=0}^{N+M}\left|h_{j, i}\right|^{2}=\theta_{1}, \ldots$, $\sum_{j=1}^{l-1} \sum_{i=(d-1)(N+M+1)}^{d(N+M+1)-1}\left|h_{j, i}\right|^{2}=\theta_{d}$. The necessary condition for the mixing parameter $\gamma$ to avoid ill convergence is given by

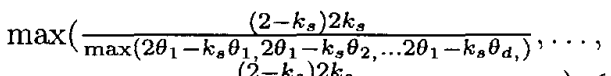

$$
\begin{aligned}
& \left.\frac{\left(2-k_{s}\right) 2 k_{s}}{\max \left(2 \theta_{l-1}-k_{s} \theta_{1}, 2 \theta_{l-1}-k_{s} \theta_{2}, \ldots 2 \theta_{l-1}-k_{s} \theta_{d,}\right)}\right) \leq \gamma< \\
& \overline{\max \left(2 \theta_{l}-k_{s} \theta_{1}, 2 \theta_{l}-k_{s} \theta_{2} \ldots 2 \theta_{l}-k_{s} \theta_{l}\right)}
\end{aligned}
$$

Since the calculation oft he desirable range for $\gamma$ requires knowledge of the combined channel + equalizer impulse response, which is difficult to estimate in practice, it would be advantageousi $f$ the lower and upper boundso $f \gamma$ are constants. Therefore, in the two-user case, which is applicable to the example of cross-polarization transmission, we assume automatic gain control (AGC) so that the reconstructed power is equalt o the transmitted power for the same time interval, i.e., $\theta_{1}+\theta_{2}=1$. By considering the worse case as that for which the retrieved sequence has equal contributions from the desired source and the interference source, i.e., $\theta_{1}=\theta_{2}$, we suggest that the mixing parameteri s chosen as $4 k_{s}$ and this is the value at which the uppera nd lowerb ound for $\gamma$ coincide. Fora multiusers ystem, an ad-hoc solution would be to use hard decision on the previous equalizer when it is estimated to give an open eye pattern and therefore the decorrelation cost becomes $\gamma \sum_{j} \sum_{\delta}\left|E\left\{y_{l}(k) \operatorname{dec}\left(y_{j}^{*}(k-\delta)\right)\right\}\right|^{2}$ after the blind startup period.

\section{SIMULATIONS}

In a QPSK system with source alphabet $\left\{ \pm \frac{1}{\sqrt{2}} \pm \frac{1}{\sqrt{2}} j\right\}$, we assume $d=2$ users, $r=3$ sensors and six random sub-channels of order $M=3$ with $20 d B$ additive white 

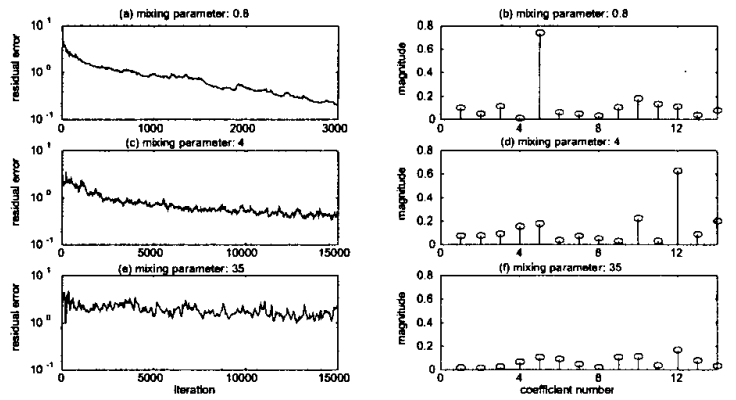

Fig. 1. The convergence of equalizer-2 in the case of undermodelling (a) Residual error, $\gamma=0.8$ (b) Undesirable convergence: combined channel + equalizer- 2 impulse response (c) Residual error, $\gamma=4$ (d) Desirable convergence: combined channel + equalizer-2 impulse response (e) Residual error, $\gamma=35$ (f) Undesirable convergence: combined channel + equalizer- 2 impulse response

noise. The order of the first equalizer is chosen as $N=3$. Since $d(M+N+1)<r(N+1)$, the channel convolution matrix is not full column rank. Indeed, this is the situation of undermodelling of channel length, and residual error is present [1] at the first equalizer output. Due to the limitation of space in the paper, equalizer-1 output is not shown. But despite the residual error, equalizer-1 retrieved source1 with delay 1. The second equalizer is also assumed to have order $N=3$. Since $\theta_{1}=0.67$ and $\theta_{2}=0.08$ with this setting, the desired bound for $\gamma$ is $1.5 \leq \kappa<24.8$ according to our analysis. In the simulation, we initialize the second equalizer to a small random value and the result is shown in fig 1 . As indicated by the combined channel + equalizer-2 impulse response, when $\gamma=0.8$ (i.e., outside the desirable range), the second equalizer converges again to source-1. When the condition on the mixing parameter is satisfied, $\gamma=4$, desirable source- 2 is retrieved. When $\gamma=35$, which exceeds the upperb ound, no source is retrieved and the equalizer converges to the origin. The mild tolerance of the lower and upper bounds between the simulation and the analysis is due to the approximation used in eq (3) and this is a subject of on-going research. In fig 2, the second equalizer is assumed to have order $N=5$. Thus the channel convolution matrix is full column rank and we have sufficient degrees of freedom to model the channel. Similar result to fig 1 is achieved.

\section{CONCLUSION}

By studying the surface characteristics of the CC-CMA algorithm, a bound for the mixing parameter $\gamma$ was obtained which prevents algorithm ill-convergence. For the two-user system, by considering the worse case, we suggest that the
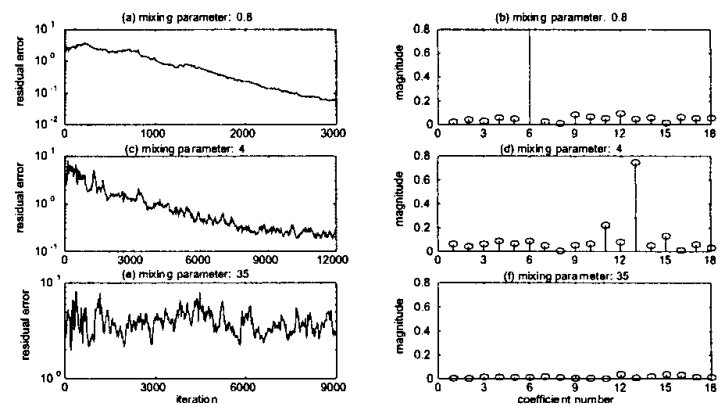

Fig. 2. The convergence of equalizer-2 in the case of sufficient degrees of freedom in channel modelling (a) Residual error, $\gamma=0.8$ (b) Undesirable convergence: combined channel + equalizer-2 impulse response (c) Residual error, $\gamma=4$ (d) Desirable convergence: combined channel + equalizer-2 impulse response (e) Residual error, $\gamma=35$ (f) Undesirable convergence: combined channel + equalizer- 2 impulse response

mixing parameter be chosen as $4 k_{s}$. Simulation studies support the analysis results.

\section{REFERENCES}

[1] C.R. Johnson Jr and B.D.O. Anderson. Godard blind equalizer error surface characteristics: White, zeromean, binary source case. In Int. J. Adaptive Control and Signal Processing, volume 9, pages 301-324, 1995.

[2] C.R. Johnson Jr, P. Schniter, T.J. Endres, J.D. Behm, D.R. Brown, and R.A. Casas. Blind equalization using the constant modulus criterion: a review. In Proc. of IEEE, volume 86(10), pages 1927-1949,1998.

[3] S. Lambotharan and J.A. Chambers. On the surface characteristics of a mixed constant modulus and crosscorrelation criterion for the blind equalization of an MIMO channel. In Signal Processing, volume 74, pages 209-216,1 998 .

[4] Y. Luo, S. Lambotharan, and J. A. Chambers. Global convergence and mixing parameter selection in the cross correlation constant modulus algorithm for the multi-user environment. In press, IEE Proceedings Vision, Image and Signal Processing,2 000.

[5] C.B. Papadias and A.J. Paulraj. A space-time constant modulus algorithm for SDMA systems. In Proc. of IEEE Vehicular TechnologyC onf., pages 86-90, Atlanta,1 996. 